\title{
Review of: "Lockdowns lose one third of their impact on mobility in a month"
}

\author{
Emre Kuşkapan ${ }^{1}$ \\ 1 Erzurum Tecnical University
}

Potential competing interests: The author(s) declared that no potential competing interests exist.

$<$ div $><$ br class="Apple-interchange-newline" $>$ I think the study is very useful for readers. After the Lackdown period, people's preference for public transportation has also changed considerably. For this reason, mobility was also affected by this situation. I recommend that readers take these situations into consideration as well.</div> 